\title{
Diseño de prototipo web inclusivo con interfaces naturales para apoyar el examen de admisión de personas con discapacidad visual en educación superior
}

\author{
Carmen Cerón, Etelvina Archundia, Beatriz Beltrán, Migliolo Jair \\ Benemérita Universidad Autónoma de Puebla, Facultad de Ciencias de la Computación, \\ Puebla, México \\ \{mceron, etelvina, beltran\}@cs.buap.mx, \\ migliolo.jair@gmail.com
}

\begin{abstract}
Resumen. En la actualidad, existe una variedad de aplicaciones de las Tecnologías de la Información y la Comunicación para el ámbito educativo. Sin embargo, se requiere atender a estudiantes con necesidades educativas especiales. El objetivo es presentar el diseño, desarrollo e implementación de un prototipo web como herramienta de inclusión para personas con discapacidad visual para apoyar la realización del examen de admisión. La metodología utilizada fue el diseño inclusivo centrado en el usuario y la implementación utiliza JavaScript y un convertidor de Texto a Voz de los archivos del repositorio. Las pruebas de funcionalidad y usabilidad se realizaron en un grupo focal de tres estudiantes con discapacidad visual.
\end{abstract}

Palabras clave: Educación inclusiva, accesibilidad, discapacidad, interfaces.

\section{Design of Inclusive Web Prototype with Natural Interfaces to Support the Admission Test for People with Visual Disabilities in higher Education}

\begin{abstract}
Currently there is a diversity of applications of Information and Communication Technologies for the educational field. However, it is necessary to attend students with special educational needs. The purpose is to present the design, development and implementation of web prototype as an alternative tool for the inclusion of students with visual disabilities, as a support for the realization of the undergraduate level admission test. The methodology used was the usercentered inclusive design and the implementation use JavaScript and a Text-tovoice converter of the repository files. Finally, the tests of the usability and functionality tests was realized with a focus group of students with visual disabilities.
\end{abstract}

Keywords. Inclusive education, accessibility, disability, interfaces. 


\section{Introducción}

La educación en México requiere atender a los estudiantes con capacidades diferentes, lo cual con lleva a generar sistemas educativos incluyentes. Los programas actuales deben ser incluyentes garantizando que los estudiantes puedan tener acceso a la educación obligatoria y superior. La educación inclusiva debe incorporar a los estudiantes con distintas discapacidades en las aulas ordinarias, lo cual implica un esfuerzo permanente.

Según los resultados del Censo de Población y Vivienda realizado por el Instituto Nacional de Estadística y Geografía [1] México tiene una población de 119,530,753 personas, de las cuales 35.2 millones están matriculadas como estudiantes en educación básica escolarizada, lo que representa $73.4 \%$ de la matrícula del sistema educativo. En educación media superior, se brinda servicio educativo a 4.4 millones de jóvenes, y en educación superior hay 3.3 millones de alumnos, lo que significa $29.2 \%$ de cobertura, distribuidos en siete mil planteles en todo el país [1]. De acuerdo a las estadísticas publicadas por la universidad solo el $10 \%$ de los alumnos en edad de ingresar a la educación superior obtiene un lugar y para las personas con discapacidad se reduce aún más en $2 \%$, ya que a pesar de los avances tecnológicos y la apertura a la educación sigue existiendo la falta de inclusión para las personas con discapacidad sin tener los materiales, tecnologías o instalaciones para apoyar el examen de admisión para obtener un lugar en las Instituciones de Educación Superior (IES)

Desde el 2008, la educación inclusiva ha formado parte de la Declaración Mundial de "Educación para Todos", como una alternativa para transformar los sistemas educativos De acuerdo con la UNESCO [2], podemos entender a la educación inclusiva como: La educación inclusiva puede ser concebida como un proceso que permite abordar y responder a la diversidad de las necesidades de todos los educandos a través de una mayor participación en el aprendizaje, las actividades culturales y comunitarias y reducir la exclusión dentro y fuera del sistema educativo. Lo anterior implica cambios y modificaciones de contenidos, enfoques, estructuras y estrategias que conlleven a lograr una educación inclusiva para favorecer a todos los actores educativos.

Para los estudiantes con discapacidades, el uso de tecnologías digitales significa principalmente dos ventajas: una mayor posibilidad y acceso a la información y el uso de recursos didácticos para poder apoyar su aprendizaje [3].

El reconocimiento automático de voz es una tecnología, que, está siendo incorporada como la interface idónea para la comunicación entre hombre y la computadora debido a la naturalidad de la comunicación que presentan los sistemas actuales de reconocimiento de voz. Estas herramientas computacionales procesan la señal de voz emitida por el ser humano y reconocen la información contenida en ésta, convirtiéndola en texto o dando órdenes que actúan sobre un proceso mediante el uso de comando.

El propósito de este trabajo es presentar el diseño de un prototipo para apoyar el examen de admisión para estudiantes con discapacidad visual usando interfaces naturales de reconocimiento de voz y el procesamiento del lenguaje natural para facilitar la evaluación. El software EDU-INCLUEXA. permite que el usuario interactúe a través de comandos de voz en cada sección de la evaluación, realizando ejercicios y simulando la Prueba de Actitud Académica, el sistema almacena los resultados de la evaluación y 
genera un archivo de resultados en formato pdf, los cuales pueden ser consultados posteriormente por el usuario y evaluador.

El artículo está organizado en la siguiente forma: en la sección 2 se presenta la revisión del trabajo relacionado con la investigación. En la sección 3 se define la metodología, el análisis y diseño de del prototipo. En la sección 4 se muestran los resultados de la prueba piloto y la evaluación para estudiantes con discapacidades en el grupo focal a través de un enfoque metodológico cualitativo y la técnica de inspección y exploración. Finalmente, se presentan las conclusiones y el trabajo futuro de la investigación.

\section{Trabajo relacionado}

En esta sección, se presentan la revisión de algunos trabajos que se encuentran relacionados con procesamiento de voz e interfaces naturales de voz utilizados para apoyar a personas con discapacidad en su vida cotidiana.

Un sistema text-to-speech (TTS) convierte el lenguaje de texto normal en habla; otros sistemas recrean la representación simbólica lingüística como transcripciones fonéticas en habla. La síntesis de texto a voz (TTS), es la conversión automática de un texto a un lenguaje nativo. El TTS, es una tecnología que permite utilizar un $\mathrm{n}$ algoritmo llamado motor TTS, que analiza el texto, procesa previamente el texto y sintetiza el texto y finalmente, genera datos de sonido en un formato de audio como salida. La calidad de un sintetizador de voz es juzgada por su similitud con la voz humana y por su capacidad de ser entendido. Un programa inteligible de texto a voz permite apoyar a personas con discapacidad visual o discapacidad lectora para escuchar documentos y poder interactuar.

En [4] la investigación se enfocó en determinar la eficacia del software de convertir "speech-text". Con el objetivo de realizar los diagnósticos y determinar si la carga cognitiva para su capacitación podría disminuir para los participantes que se clasificaron en tres discapacidades categóricas que afectan las áreas de lectura y expresión escrita. Para lo cual analizaron muestras de escritura de los estudiantes con y sin apoyo del software, verificando que los participantes mejoraban en relación a los que utilizaron el software. El software que utilizaron fue aplicaciones en plataformas de iOS y Windows.

En [5] el trabajo presentado se enfoca al uso de software TTS para mejorar las habilidades de lectura para estudiantes con dificultades de rendimiento académico. El software TTS utilizado para este estudio fue el Kurzweil 3000, que fue diseñado como una herramienta compensatoria para personas con discapacidades, incluidas discapacidades de aprendizaje (LD), trastorno por déficit de atención con hiperactividad (TDAH), dificultades de lectura y algunas discapacidades físicas, como la tetraplejia. El software TTS proporciona una presentación visual y auditiva sincronizada de texto. Reproduce texto mediante palabras habladas de documento electrónicos. Finalmente, los resultados demostraron que los participantes que utilizaron el software TTS habían mejorado en la lectura, el vocabulario y comprensión. 
En la investigación realizada en [6], diseñaron un software para la conversión de texto a voz para personas con discapacidades visuales. El desarrollo del sintetizador de texto a voz en forma de una aplicación simple, convierte el texto ingresado en voz sintetizada y posteriormente se lee al usuario, el cual puede guardarse como un archivo mp3. Logrando obtener un motor para el idioma ingles y posteriormente para el nigeriano.

Con respecto a las Interfaces de Usuario Natural (NUI), los investigadores se centran en la creación y evaluación de gestos y acciones naturales realizados para una interacción multitáctil.

En [7] afirman que las NUI, son consideradas nuevos métodos para la Interacción Humano Computador (HCI) y el diseño de aplicaciones informáticas basadas en interfaces con las cuales las interacciones se realizan a partir de las acciones naturales de los seres humano, es decir, que permiten reconsiderar las habilidades existentes del usuario. Por lo cual, se consideran bastantes adaptables y accesibles para las personas con alguna discapacidad. Por otra parte, afirman que las características de las NUI son centradas en el usuario (necesidades, deseos y limitaciones), multicanal (habilidades sensoriales y motoras), extendidas, de gran ancho de banda, y de interacción basada en voz (procesamiento de voz y comunicación), basadas en imagen (integran imágenes para la comunicación del usuario), basadas en comportamiento (reconocimiento del comportamiento humano y de expresiones) logrando apoyar a los usuarios en sus necesidades.

El reconocimiento de voz o Automatic Speech Recognition (ASR) [8] es el proceso de convertir una señal de voz a una secuencia de palabras mediante un algoritmo, implementado como un programa de computadora [9]. Las interfaces son mecanismos diseñados para permitir el control de un dispositivo o aplicación mediante la interacción verbal. El reconocimiento de voz es generalmente utilizado como una interfaz humanocomputadora usado por un software de aplicación, el cual debe cumplir tres tareas

1. Procesamiento: Convierte la entrada de voz a una forma que el reconocedor pueda procesar, es decir, convertir la señal análoga a digital.

2. Reconocimiento: Identifica lo que se dijo, realizando la traducción.

3. Comunicación: Envía el reconocimiento al software de aplicación.

Tradicionalmente, las tres áreas principales de trabajo en el campo del procesamiento de voz desde un punto de vista del procesamiento de señales son: codificación, síntesis y reconocimiento. Lo cual en [10] y [11] demuestran que la síntesis de texto a voz es una tecnología computacional de rápido crecimiento y desempeña un papel cada vez más importante en la forma en que los usuarios interactúan con el sistema y las interfaces logran integrarse a una variedad de plataformas y adaptarse a las necesidades de los usuarios.

\section{Metodología}

La metodología que se utilizó fue un enfoque cualitativo, usando el Diseño Centrado en el Usuario Inclusivo (DCUI) y el modelo de Prototipos, el cual se describe en el análisis y diseño del sistema. 


\subsection{Análisis y diseño del prototipo}

El DCU inclusivo se basa en los criterios de usabilidad de heurística [12] y en las líneas de la Guía de Accesibilidad al Contenido en la Web. Por lo cual se aplicaron los criterios de: uso de colores, independencia del dispositivo, la navegación mediante comandos de voz. Así también, se realizó una serie de entrevistas y encuestas con el grupo focal, logrando identificar las necesidades de interacción. Para el análisis y el diseño del sistema se determinaron los diagramas de secuencia, como se muestra en Fig. 1. El sistema permite identificar dos usuarios para el acceso y manipulación del sistema:

- Usuario Aplicador: Puede realizar consultas generales de la evaluación del tema y examen

- Usuario Aspirante con baja visión/Ceguera: siendo el cual elije el tipo de evaluación: Prueba de Aptitud Académica (PAA) o Prueba por Área de Conocimiento (PAC). Además, el software incluye una serie de actividades de ejercicios para cada una de las pruebas como una guía de ayuda antes de aplicar la prueba y finalmente se le muestra su resumen de la evaluación.

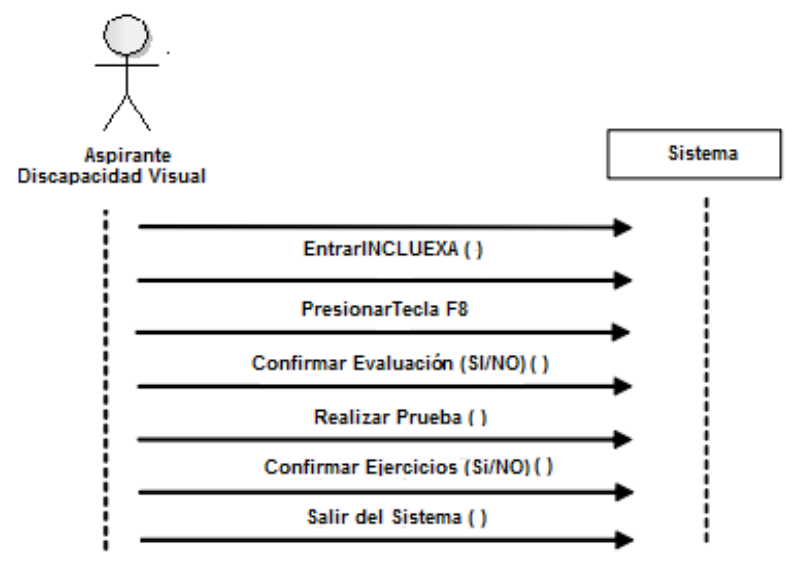

Fig. 1. Diagrama de caso de uso del estudiante.

Así también, se utiliza un dispositivo natural que se encarga de recibir la voz del usuario (la entrada) y posteriormente se encarga de procesar la voz del usuario y ejecutar la función correspondiente (procesamiento de voz) por medio una función lambda que se encarga de interpretar los comandos de voz del usuario, y convertirla en acciones que la API entienda por medio de la libreria Artyom.js en JavaScript. Tal como se muestra en la Fig. 2 las acciones e interacción con el software. 


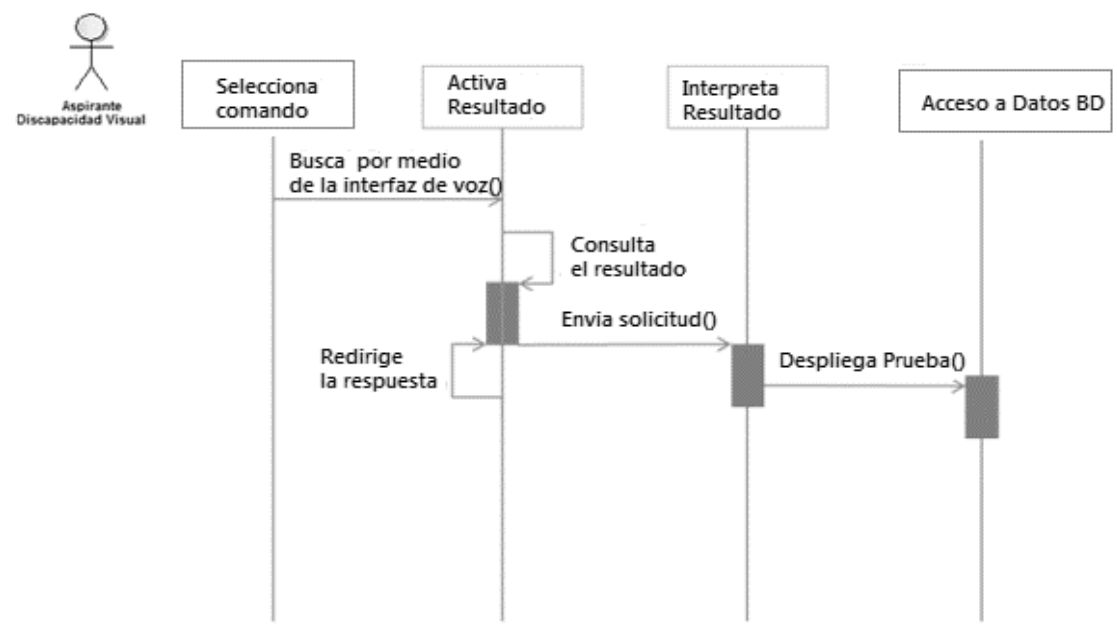

Fig. 2. Diagrama de interacción del usuario con el software.

\subsection{Arquitectura y desarrollo del prototipo}

El Sistema de reconocimiento de voz, maneja un extractor de características y el clasificador. Cuando se recibe la señal de voz, se pasa a través de un reconocedor que da como resultado la palabra. Después de un procesamiento de lenguaje natural, se realiza una representación semántica y finalmente una acción. Hay dos procesos importantes en la fase de reconocimiento. La extracción de características tiene los siguientes pasos:

- La señal se divide en una colección de segmentos.

- La técnica de procesamiento de señales se utilizará para obtener una representación de las características más distintivas del segmento.

- Basado en sus propias características, se construyen un conjunto de vectores que constituyen la entrada al siguiente módulo.

En cuanto al clasificador probabilístico, tenemos lo siguiente:

Un modelo probabilístico basado en redes neuronales se crea como modelos ocultos de Markov y con la probabilidad de que esto ocurra, se realiza una búsqueda para encontrar la secuencia de segmentos con la mayor probabilidad de ser reconocido.

La estructura del sintetizador de texto a voz se puede dividir en módulos principales:

a) Módulo de Procesamiento de Lenguaje Natural (NLP): Produce una transcripción fonética del texto leído, junto con la fonética.

b) Módulo de procesamiento de señal digital (DSP): transforma la información simbólica que recibe de la PNL en voz audible e inteligible. 


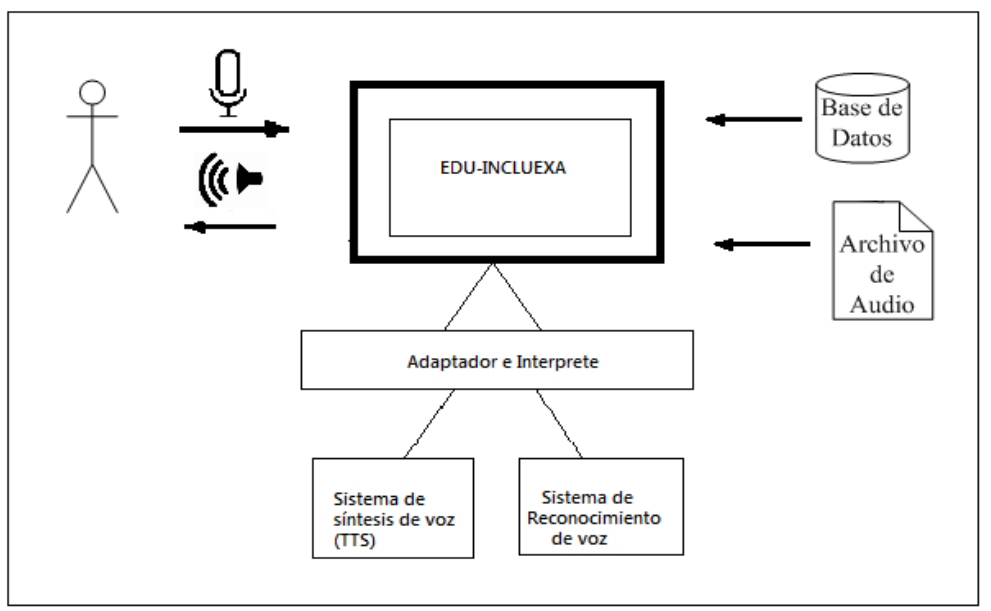

Fig. 3. Arquitectura del sistema.

La biblioteca utilizada en lenguaje JavaScript para el reconocimiento y síntesis de voz fue Artyom.js, que se basa en el "web-kit-speech-recognition". Esta biblioteca tiene soporte en español e inglés, por lo que el reconocimiento de voz y el uso de la plataforma se pueden realizar en ambos idiomas. El diseño del prototipo se muestra en la Figura 4, las forma de interactuar, la lista de tareas y configuración de acuerdo a las necesidades del usuario.

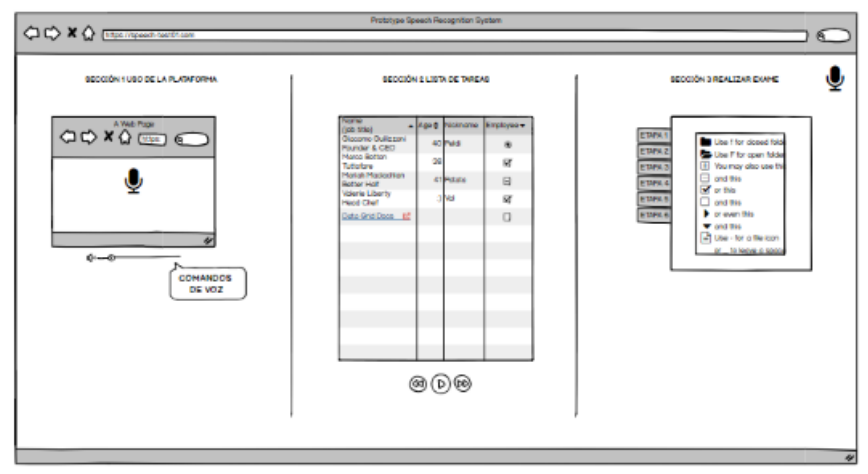

Fig. 4. Prototipo web inclusivo.

El desarrollo del software fue por prototipado, lo cual permitió trabajar con los usuarios. Para la implementación se utilizando HTML, CSS y JavaScript, accediendo directamente a las API de reconocimiento de voz y a la biblioteca Artyom.js. Del mismo modo, se hace uso del reconocimiento de comandos del teclado, de modo que el usuario puede usar algunas combinaciones de teclas para acceder a la plataforma, como se muestra en la Figura 5. 


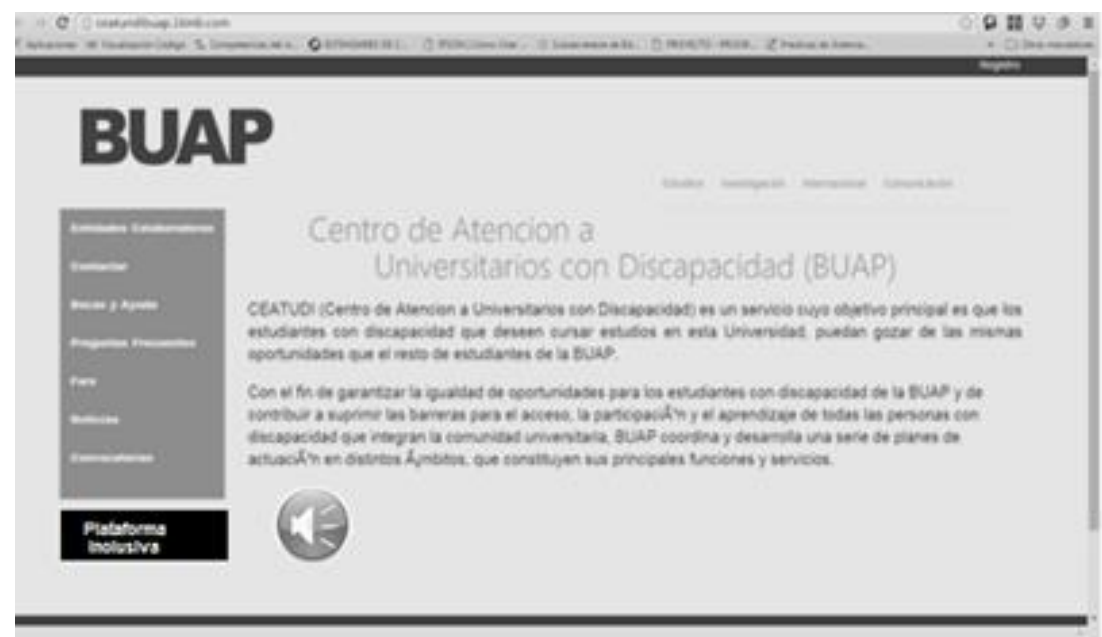

Fig. 5. Ingreso al sistema "EDU-INCLUEXA".

Después de la pantalla de inicio de sesión, el usuario tiene la posibilidad de navegar por la plataforma, ya sea entre las secciones para la preparación del examen y aplicación de prueba de examen. En la Figura 6 se visualiza la pantalla que presenta las instrucciones para poder contestar las preguntas.

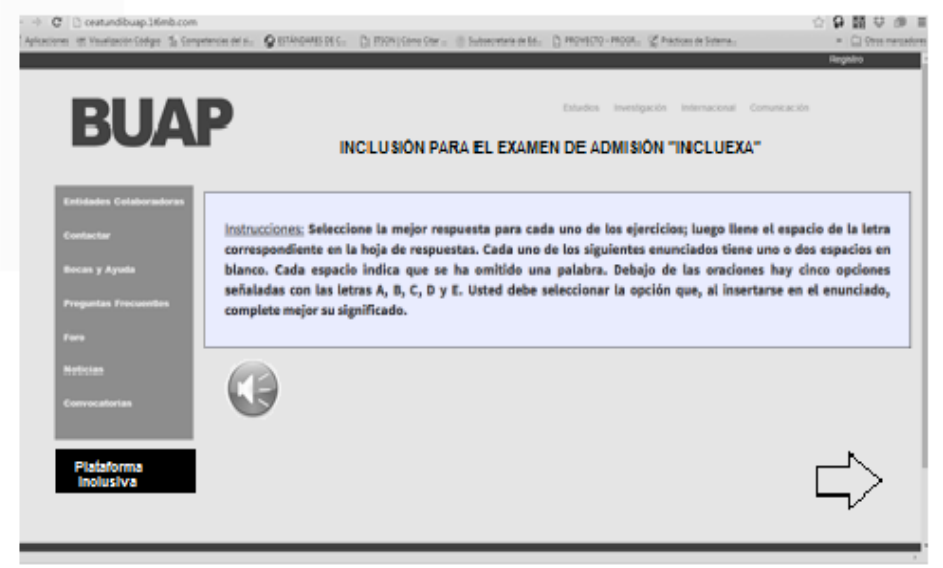

Fig. 6. Pantalla de instrucciones para navegar en la evaluación.

A partir de las instrucciones el usuario con discapacidad visual puede interactuar con voz o teclado para que pueda contestar las preguntas seleccionando la respuesta correcta como se muestra en la Figura 7. 


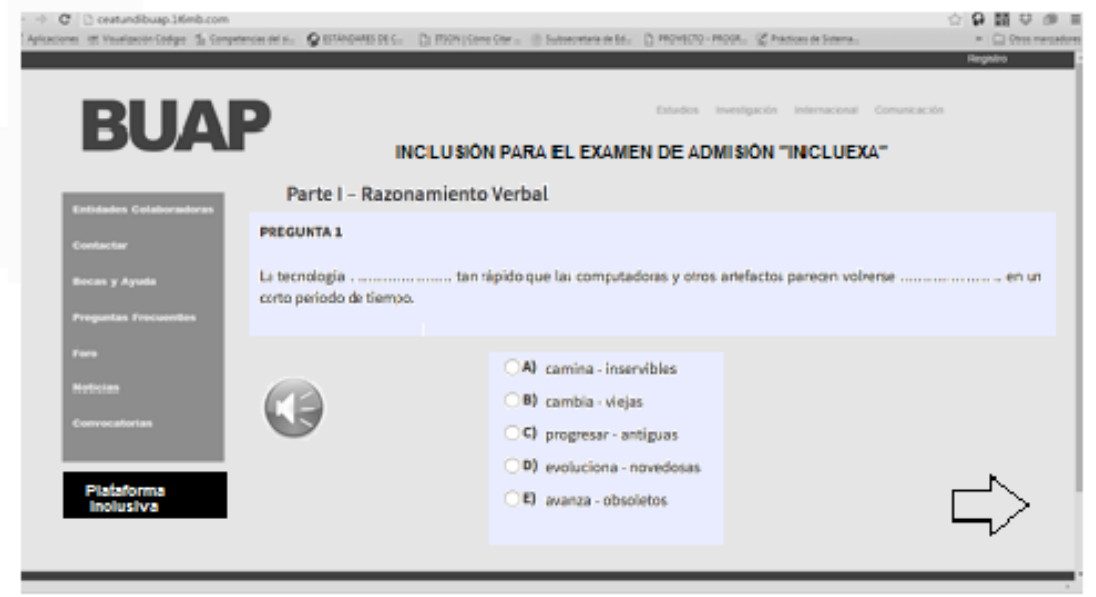

Fig. 7. Pantalla de selección de respuesta por el usuario.

Así también para el seguimiento de su aprendizaje, se le proporciona los resultados en pantalla, se almacena y se genera un archivo en formato pdf, que posteriormente puede consultar el estudiante o evaluador (ver Figura 8).

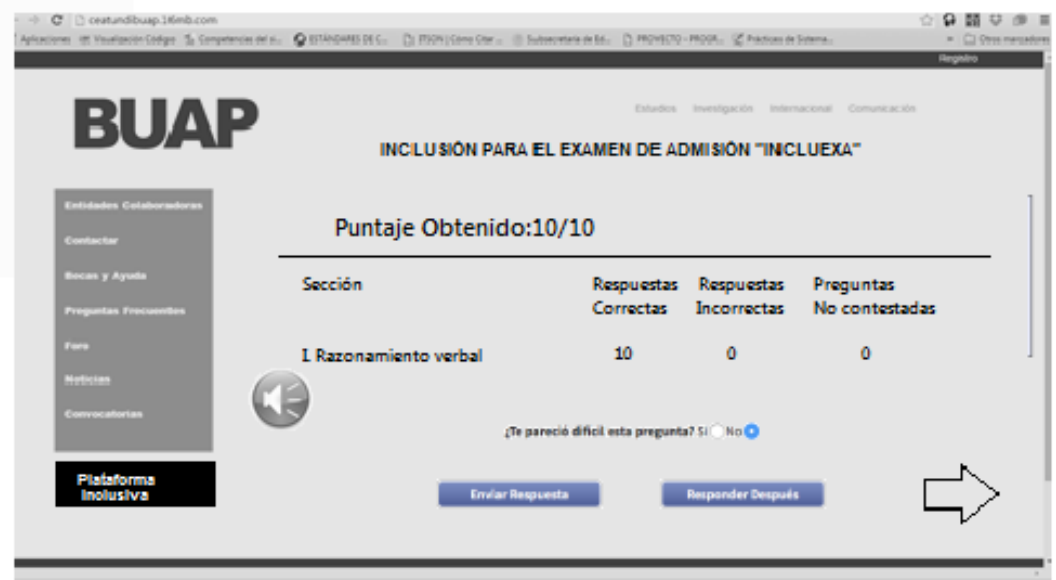

Fig. 8. Pantalla de resultados del estudiante.

\section{Resultados}

El prototipo web inclusivo se integró al portal del Centro de Atención a Universitarios con Discapacidad. La prueba funcional del prototipo se llevó a cabo con un grupo focal conformado por dos alumnos con disminución de discapacidad visual, y una alumna con ceguera, los cuales realizaron los ejercicios y pruebas. 
Para lo cual, se aplicó la técnica de inspección y exploración bajo tres posibles escenarios que a continuación se describen:

- Situación 1: Al usuario se le dio una breve explicación del uso del sistema y se le acompaño en el sistema en su navegación y ejecución de la evaluación.

- Situación 2: Al usuario se le explicó el uso del sistema y solo se le acompañó al inicio de la evaluación.

- Situación 3: Al usuario se le explico el uso del sistema y no se le acompañó en la navegación ni ejecución de las actividades.

Los usuarios deberán cumplir ciertas tareas para comprobar el funcionamiento del sistema:

- Tarea 1: Acceder al sistema registrando su clave y contraseña, seleccionando el control por voz, comandos o hipervínculos de imágenes.

- Tarea 2: Seleccionar del menú de navegación: Ejercicios de Prueba o Simulador de Aplicación de Prueba de Aptitud Académica con formada por secciones para Lectura, Redacción, Matemáticas e inglés.

- Tarea 3: Recorrer el sistema mediante la interacción seleccionada voz, comandos de teclas o hipervínculos de imágenes.

- Tarea 4: Obtener el resultado de avance de ejercicios y de la aplicación del simulador de la Prueba de Aptitud Académica y generar la evaluación en un archivo.

Los resultados obtenidos de la prueba de inspección se presentan en la Tabla 1. Lo cual refleja que los usuarios con una breve explicación de la Situación 1 su desempeño fue del $87.58 \%$ del cumplimiento de las tareas mientras que los usuarios de la Situación 2 al $82.5 \%$ y para la situación 3 lograron las tareas solo en un $80.5 \%$ esto implica que el software inclusivo apoya la ejercitación para la preparación y simulación del examen de admisión de manera que las interfaces de voz facilitan la interacción con el software a las personas con discapacidad visual.

Tabla 1. Resultados de la Prueba funcional por inspección.

\begin{tabular}{cccc}
\hline Tarea & Situación 1 & Situación 2 & Situación 3 \\
\hline 1 & $90 \%$ & $85 \%$ & $76 \%$ \\
2 & $90 \%$ & $80 \%$ & $85 \%$ \\
3 & $85 \%$ & $85 \%$ & $80 \%$ \\
4 & $85 \%$ & $80 \%$ & $80 \%$ \\
$\mathbf{M =}$ & $87.5 \%$ & $82.5 \%$ & $80.25 \%$ \\
\hline
\end{tabular}

Esto implica, que el campo del uso de las interfaces de voz debe ser considerado para el diseño de software inclusivo y brindar mayor accesibilidad a las personas que tiene necesidades especiales para la educación. 


\section{Conclusión y trabajo a futuro}

Una de las principales contribuciones, es la aportación de un prototipo de software inclusivo para apoyar a la preparación del examen de admisión a las personas con discapacidad visual logrando integrar las tecnologías de reconocimiento de voz para facilitar la interacción del usuario y el uso de las interfaces naturales.

Este trabajo investigación, cuya finalidad principal fue brindar un apoyo tecnológico para motivar a estudiantes con discapacidad visual para continuar sus estudios a nivel superior y promover la preparación para presentar el examen de admisión para ingresar a las Instituciones de Educación Superior. Dando pauta hacia un nuevo panorama en la educación superior inclusiva apoyado por las tecnologías de voz e interfaces naturales de usuario las cuales brindan una mejor asistencia e interacción con las personas con discapacidad y permiten reducir la brecha digital.

Como perspectiva de este trabajo, se pretende generar más reactivos para la base de datos que apoyen a la Prueba de Aptitud Académica e incorporar la Prueba de Área de Conocimiento. Así como mejorar las interfaces y operatividad del sistema, De tal manera que se pueda constituir una alternativa para presentar el examen de admisión en la educación media superior y superior.

Por otra parte, las pruebas de accesibilidad y usabilidad deben aplicarse a una mayor muestra, para que se obtengan estadísticas sobre el uso del software inclusivo en estudiantes con alguna discapacidad y generar otros apoyos de recursos digitales y herramientas para el aprendizaje de estudiantes en educación superior.

\section{Referencias}

1. Instituto Nacional de Estadística y Geografía (INEGI). Censo de Población y Vivienda (2015). Recuperado de http://www.beta.inegi.org.mx/temas/estructura/

2. UNESCO: Directrices sobre políticas de inclusión en la educación. http:// unesdoc.unesco.org/images/0017/001778/177849s.pdf, último acceso 2017/11/09

3. Fernández, J.: Necesidades educativas especiales en el contexto universitario español. Revista de la Educación Superior, 33, 149-162 (2004)

4. McCollum, D., Nation, S., Gunn, S.: The Effects of a Speech-to-Text Software Application on Written Expression for Students with Various Disabilities. National Forum of Special Education Journal, 25, 1-13 (2014)

5. Stoddena, R., Robertsa, K., Takahashia K., Parka, H., Stoddena, J.: Use of text-to-speech software to improve reading skills of high school struggling readers. Procedia Computer Science, 14, 359-362 (2012)

6. Isewon, I., Oyelade, J., Olufunke, O.: Design and Implementation of Text to Speech Conversion for Visually Impaired People, International Journal of Applied Information Systems Foundation of Computer Science FCS, New York, USA, 7(2), 25-30 (2014)

7. Lozada, R, Rivera, L., Molina, F.: Interfaces de Usuario Natural. https://www.researchgate. net/publication/285927377_Interfaces_de_Usuario_Natural,último acceso 2017/10/09

8. Zheng, H., Haiyan, H., Shihong, L., Hong, M.: The Study and Implementation of TextSpeech System for Agricultural Information. In Third IFIP TC 12 International Conference on Computer and Computing Technologies in Agriculture III (CCTA), Oct 2009, Beijing, 
Carmen Cerón, Etelvina Archundia, Beatriz Beltrán, Migliolo Jair

China. Springer, IFIP Advances in Information and Communication Technology, AICT317, 291-296 (2010)

9. Sung, J., Kyun, D., Shin, Y.: Design and Implementation of a Voice Based Navigation for Visually Impaired Persons. International Journal of Bio-Science and Bio-Technology, 5(3),61-68 (2013)

10. Lleida, E: Reconocimiento automático del habla. Centro Politécnico Superior Universidad de Zaragoza. Recuperado de http://www.gtc.cps.unizar.es/ eduardo, último acceso 2017/10/22

11. Newell, A., Gregor, P.: User Sensitive Inclusive Design: in search of a new paradigm. In: CUU First ACM Conference on Universal Usability (2000)

12. Nielsen, J: Ten Usability Heuristics, http://www.useit.com/papers/heuristic/heuristic_list.html, último acceso 2018/01/25 